December 21.-Patient discharged from hospital. No return of spasms. Crossness and viciousness very much improved.

Nay 10, 1893.-Reports from patient indicate marked improvement in his mental condition. Language is being acquired with great rapidity. Education already begun with gratifying success. No return of convulsions since discharge from the hospital.

This was a typical case of idiocy due to convulsive seizures, I believe, and may be taken as an illustrative case of what can be accomplished by operative procedures in cases of suitable character. Of course no man can make a diagnosis of angioma of the dura before operation, so that in a measure such cases are exploratory. Although too little time has elapsed since the operation to speak definitely as to the ultimate result, it seems to me that the amount of good already accomplished more than compensates for the outlay of time and money.

(D.) Cerebral Softening.-It was my good pleasure to report the first case of trephining that was ever made for softening of the brain. By the term "softening" I do not mean cerebral abscess; all surgeons would trephine for that disease; nor to the condition formerly called "red softening," which is in reality only the initial stage of a suppurative inflammation, which, if not arrested, leads to destruction of the nerve cells by pyogenic microörganisms-but to the condition which follows cerebral hemorrhage or embolism, in which, upon opening the skull, we find a softened mass semi-fluid in consistence, of purulent appearance, but in reality not pus. The case mentioned was one of cerebral hemorrhage involving the speech center and the motor area of many months' standing. The diagnosis was clear and unmistakable-the maddening sensations in the head and the temporary fits of irritability in which attempts had been made upon the life of his wife were undoubtedly due to a condition of true softening. The head was opened under the strictest antiseptic precautions, and a cavity containing nearly a pint of broken-down brain and other debris (but no pus) was carefully irrigated. Thiscavity was filled with normal salt solution, the dura replaced and stitched, the scalp sewed into place without drainage, with a firm compress of gauze. His recovery was uneventful as already recorded elsewhere; nor was the improvement merely temporary as was predicted by some who heard for the first time my proposition to relieve such cases by operation. To this day he has had no recurrence of the bad symptoms. He is enjoying life in traveling, and has sent me a number of other cases for trephining. He feels that his improvement has been decidedly great, and he is going about, spreading the good news that for such hitherto helpless cases there is a possibility of relief in surgical interference.

Thyroid Feeding in Myxedema.-Recent observations show that feeding of the thyroid gland is quite as efficient in the treatment of myxedematous conditions as the hypodermatic injection of the thyroid extract, while it is much less troublesome and is free from the grave risks attendant upon the injections.

Alexander (Medical Chronicle, No. 3, p. 175) reports the entire disappearance of a myxedema lasting two years in an insane woman. The mental condition was not improved. Half of a thyroid gland of a cow was employed, given twice weekly, finely divided and mixed with bread crumps.
INTESTINAL ANASTOMOSIS-BY A NEW

METHOD, WITHOUT PLATES AND WITH

BUT TWO KNOTS-EITHER SILK

OR CATGUT SUTURES MAY

BE USED.

Read before the Section of surgery aud Anatomy at the Forty-fourth Annual Meeting of the American Medical Association. BY M. E. CONNELI, M. D.

SUPERINTENDENT OF MILWAUKEE COUNTY HOSPITAL.

When through injury or disease it is found necessary to exclude or remove a portion of the intestinal canal, the question of restoration of its continuity which comes to the surgeon, and how or which is the best method to accomplish this desired result is one of great importance.

Many methods are now in the surgical field; but we caunot have one too many if all are good, and the surgeon must familiarize himself with one method or be equally proficient in all, in case of an emergency, such as gunshot wounds or strangulated hernia.

The universal interest which has been manifested in the plate operation and its modifications, indicate very clearly that any method which promises an improvement in the technique of intestinal surgery, whether in suturing or the saving of time, will be gladly welcomed by the profession.

It is now more than a year ago that I made a union, which seemed to me to promise a great deal; a method which I thought could be applied to the intestinal canal at almost any point, and wherein the number of knots would be reduced to two. I do not wish to be understood as claiming that a reduction in the number of knots would in itself be any improvement; while of course it would be a factor very acceptable to the surgeon - if time could be saved in this manner, and there would be a coalescence of the other points in the technique, thus forming a desirable combination with the number of knots.

It is obvious in the method I will endeavor to describe to you, and I think you will agree with me, that more than two knots would be detrimental; one tie at each end of the incision or union is all that can be used advantageously, for the one great desideratum connected with this method is that every part of the suture be buried out of sight, as in the plate operation, and where the number of knots is increased to three, four, six or more, I do not see how this very important point could be achieved, for you will notice that if this suture is made properly, knots at any point other than the end could not be securely buried.

The idea herein involved is probably a modification of the plate operation, that is, the same in principle but without plates.

I felt certain that if I could penetrate or transfix both bowel walls when the serous serfaces were in apposition, and after being transfixed could keep them so, that is, with the serous surfaces in constant contact I would accomplish by means of this suture what had been gained by the plate union.

With this object in view I tried a series of experiments on dogs. I placed the serous surfaces of the walls to be sutured in apposition, and secured them so by a continuous to and fro or serpentine suture. I then drew these two walls apart, loosening the suture, so to speak, and turning the intestine over 
brought the serous surfaces of the other two walls together, and transfixed them with a similar suture.

The results have indeed been remarkable. This idea I have modified so as to apply it to all parts of the intestine, to the stomach and gall bladder as well.

It would only consume your valuable time to detail the many attempts and failures which led up to this method of suturing, which I do not doubt will recommend itself to you as eminently fitting for the work that I claim it will do.

My first application of this suture was in oblique enterorrhaphy; the intestine being divided obliquely a la Robert Morris of New York; but my effort at this session will be to briefly describe to you this method of suturing as applied in anastomosis, wherein the initial steps are the same as in the plate operation.

The bowel is severed or a portion resected; the cut ends of which are then invaginated and closed with catgut or silk sutures.

The closed ends are passed by each other, and an opening (I prefer one slightly diamond, or oval shaped), of any length desired is then made in the convex border of both the proxinal and distal portion of intestine.

The opposing walls are then placed side by side, so that the ends of incision are parallel with each other, and I found that it facilitates the work to have a suspending thread or loop run through the bowel at each end of the opening, and held by an assistant. To insert this thread for suspension, you pass your needle from within outwards through the walls of one portion of bowel, and over to the other, passing the needle from without inwards, then bring both ends up through the openings and tie, thus having the knots above. (See A.)

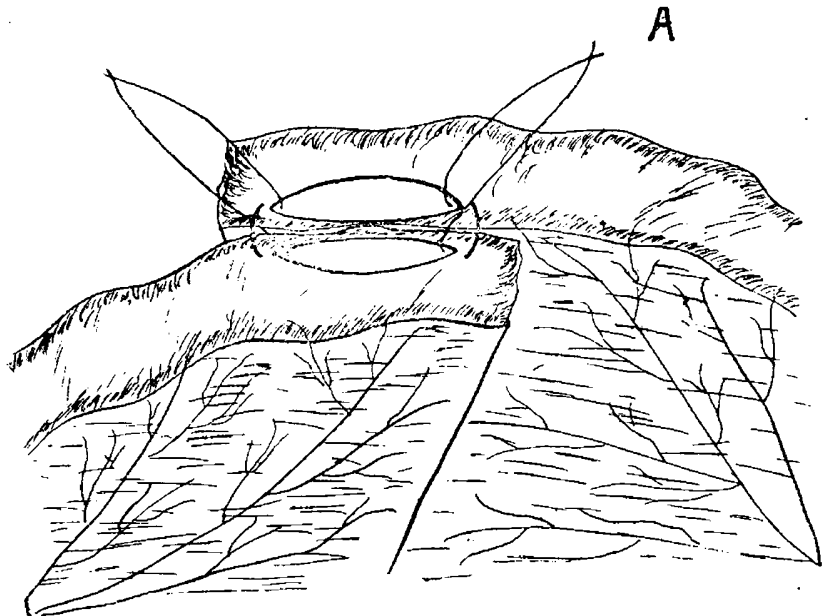

When there is a tendency to great eversion of the margins of the openings, or when you have made an unusually long incision, it will further greatly assist you to have a third suspending thread inserted midway between the other two.

As your assistant applies gentle tension or traction to these suspending loops, the opposing serous surfaces are brought into perfect contact, and you can very quickly insert the first suture, which is of looped stitches, by inserting your needle from without inwards through the bowel wall, at the right end of one opening, then passing it back and forth through all coats of both walls which are in apposition until the full length of incision has been secured, and pass your needle from within outward through the bowel wall at the opposite end of opening, leaving

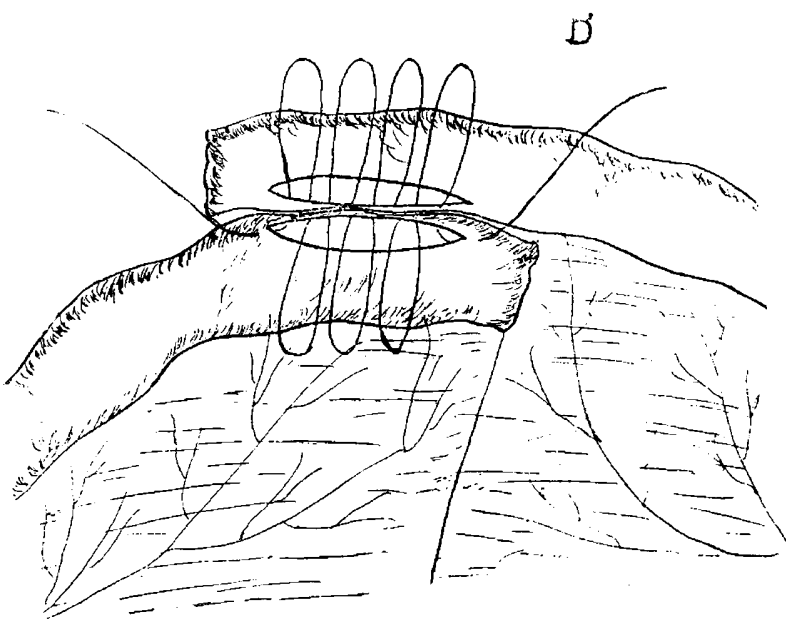

each stitch as a loop on the side where taken, (as in B) or each alternate stitch may be loose, thus having your loops all one side, (as shown in C).

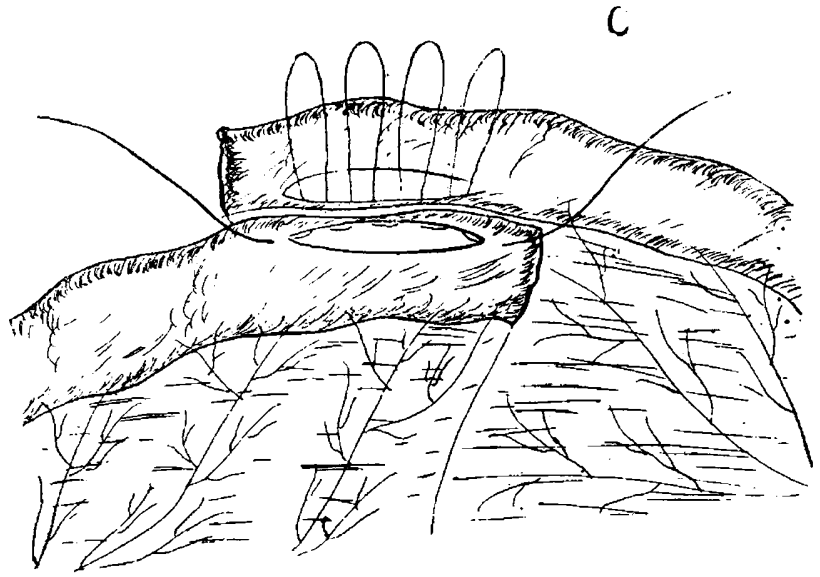

You next withdraw the middle suspending loop, if you have inserted one, and then separate these sutured walls as far as the looped stitches will allow; pass the knotted ends of suspending loops down

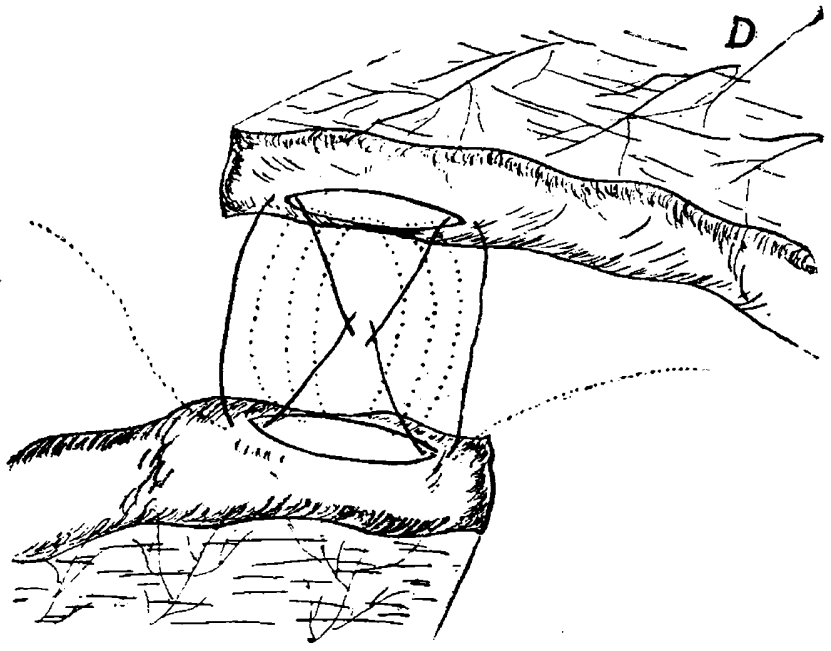

through the middle, (D) draw them up on the other side, and you will have the serous surfaces of the other two opposing walls in opposition. (See E.) 
You can now put in a middle suspending thread if you wish, and then insert your second suture, in the same manner as the first, with the exception that no loops are made, all stitches being drawn tight.

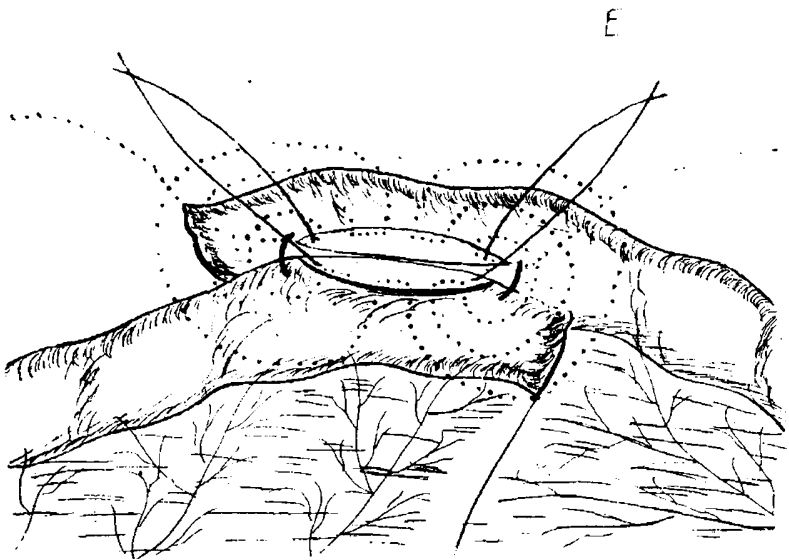

You now withdraw all suspending threads and draw up the loops of your first suture, and pull the opposing ends of both sutures till taut, and tie; not cutting off the ends of sutures after making the first knot until you have made the second, as they are necessary in making traction.

After both knots are tied, cut off the ends close, so as to leave no dangling drains, and your union by anastomosis is complete. (See F.) It has never been found necessary to use any retaining sutures.

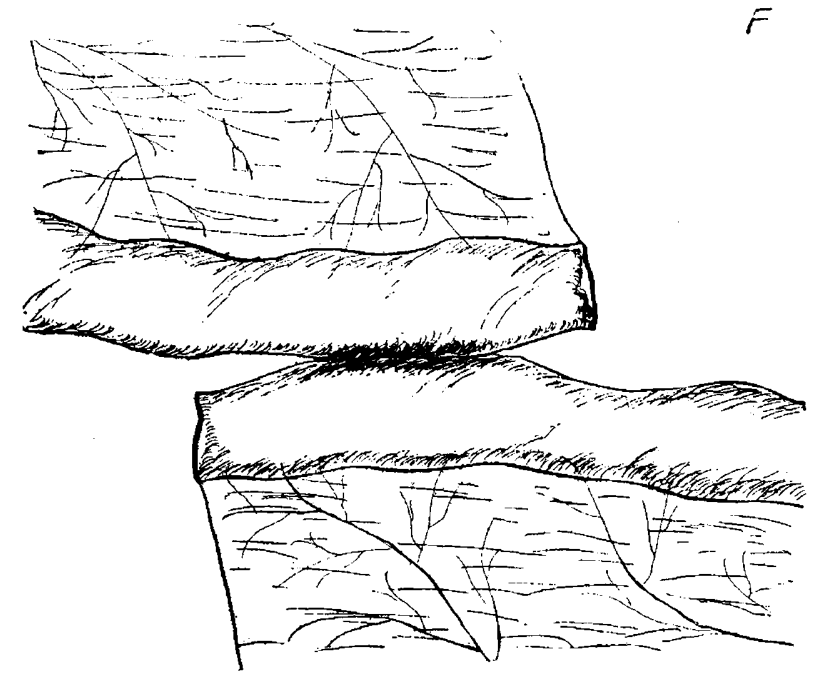

When using catgut for suturing, the knot must be secured with a silk ligature to prevent loosening, as it becomes softened by the intestinal and serous fluids.

This same result is achieved by making your first suture of tight stitches, in which case you will avoid the turning over of the intestine, which seems somewhat puzzling to not a few surgeons and then your second suture is inserted as shown in illustration G.

By inserting your needle from without inwards as before, and bring it out on the same side at about a distance of $1 \frac{1}{4}$ of an inch, then passing it over to the opposite wall and take a stitch through all coats about $1 \frac{1}{4}$ of an inch long, parallel with the margin of wound, and repeat until all has been secured and pass your needle out as before, when your sutures are drawn taut and tied and you have same result as at $\mathrm{F}$.
In the series of experiments to which I have referred, many specimens were preserved, and from them I have selected these, which you will see are illustrative of the results obtained from the application of this suture in anastomosis.

I have made no attempt to dissect or remove any of the stitches or suturing material, and as I am aware that time is a great factor in proving the value of a method, in some cases I have allowed quite a lengthened period to elapse between the experiment and the resection or death of the animal, the shortest time having been five days and the longest seven months and eight days; there is one still living of eight months duration.

In oblique enterorrhaphy the shortest time was fourteen days and the longest five months and twelve days; there is one still living of eleven months duration.

In circular union where I employed but one knot, the shortest time elapsing between experiment and resection, was twelve days, and there are three living, two of about eight and a half months duration and one where three circular unions were made at one time, which are now of six months and eighteen days duration.

The date of the experiment and time of resection or death of the animal is tagged with each specimen.

Although it may seem a little foreign to the subject of anastomosis, still I wish to mention and show you the specimen of No. 10 which operation was made on July 14th, 1892, when a union in two places at one sitting was made; both of these unions were of the oblique method and about one foot apart.

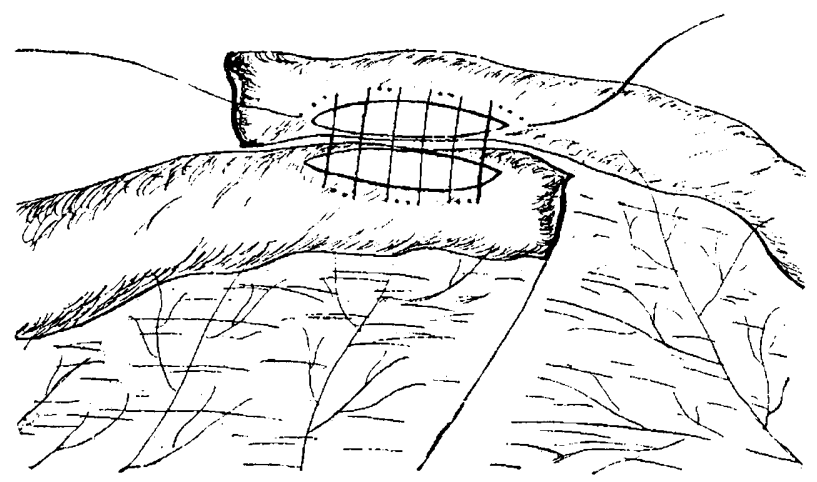

The subject was a large mastiff, the suture material very heavy catgut with darning needles. On August 29th, 1892, one (1) month and fifteen (15) days after the operation was made, I concluded to kill the animal and remove the specimen, so no effort was made toward asepsis or antisepsis and before resecting I also thought that I would practice on the intestine. An oblique enterorrhaphy was made between the two unions before mentioned. Then I resected that portion of intestine embracing these three unions and reunited the divided intestine by anastomosis, using the looped and tight stitch suture. Three suspending loops were used with perfect ease.

This anastomotic union was also made only for practice, but it looked so perfect that I concluded to close the abdomen and give the animal a chance to live, thus making experiment No. 17.

The first to which I wish to call your attention is that of experiment No.12. It was on intestinal 
anastomosis, the experimental operation being made August 1st, 1892, in the presence of Dr. J. H. McBride of the Milwaukee Sanitarium, the subject being a small pug dog.

This was the first anastomosis made by this suture, my thought in previous work having been in the direction of an end to end union by the oblique incision a la "Morris."

In this instance the first suture was of tight stitches, and the second was loose or parallel, though not looped stitches.

The dog was killed August 16th, fifteen (15) days after the operation. There were no adhesions, the abdominal cavity being free from all signs of any inflammation, the internal organs lying free and in normal position. The intestinal union is perfect; there seems to be no cicatricial contraction worthy of mention.

I would next like to have you observe the specimen of No. 13 .

In this case there was a double operation made on August 3rd, 1892. An anastomosis similar to that of No.12, which we have just examined, and an oblique union.

This subject was a very large savage bulldog which was given to me as a sacrifice to science on account of his being so very troublesome. He escaped from our kennel fifteen days after the operation and when we went to his former home for him, his master would not give him up, stating that the operation had changed his disposition to that extent, that the family were willing to give him a new trial.

The dog's good behavior continued for several months, when he slid back into his old habits, and he was again turned over to us and was killed March 21st, 1893, seven (7) months and eighteen (18) days after the operation.

Silk sutures were used in both the anastomosis and the oblique enterorrhaphy.

The specimens will speak for themselves. No adhesions, or signs of any inflammatory action were found.

The next specimen of interest is that of No. 14, which in all probability can be considered under the head of anastomosis, it being that of a gastro-enterostomy, the union of the stomach at the convex surface with the duodenum at about its middle third.

The operation was made on August 10th, 1892, and the animal killed September 22nd, 1892, twelve days after the operation. Here also there was found no adhesions of abdominal contents. FF silk suture was used in making this union which seems perfect in every respect. (The communicating foramen being quite large.)

One month and eighteen days after the second operation was made, the animal was killed and we have here a very interesting specimen. It is extremely valuable in showing how nature will come to the surgeon's rescue, if given half a chance.

Here we have an anastomosis with what would be deemed an extraordinary length of incision, namely one and one-half inch, and following even this unusually long incision we still find the formation of an enterolith, with which as it increases in size from accumulations we find the incision or anastomotic opening and the bowel enlarging from day to day, so that in one month and eighteen days after the anastomosis was made we have the proximalend of the intestine distended to a distance of about ten
(10) inches from the point of operation. Yes, distended we might say to four times its normal size and the distal portion of the bowel is also enormously dilated.

This portion of intestine in its distended state, produced by the obstruction from the enterolith, resembles in point of capacity that of an ordinary dog's stomach. It may be of interest to note here that at the time of making the anastomosis, instead of placing the ends of divided bowel so that the peristaltic action would be downward, they were so placed that both closed ends were parallel, thus directing the peristaltic wave of the distal portion upward, which no doubt favored the formation of the enterolith considering the mixed diet which a dog selects.

The specimen of No. 18 is that of an intestinal anastomosis made in the presence of a "doubting Thomas," Dr. Mackie, after his return from his last visit to Europe. The operation was made September 6 th, 1892, and the animal killed one month later, on October 6th, 1892. No adhesions were found and union is perfect.

In No. 21 the subject was a large white dog. On September 28th, 1892 a double anastomosis was made in the presence of Dr. Mackie of Milwaukee and Dr. Philler of Waukesha. The animal was killed October 6 th, 1892, ejght days after the operation; and at the site of the first union an immense enterolith was found. This specimen is of interest in showing, that while both unions were made at the same time, the concretion of bone had formed at the site of the proximal union only, while reparative adhesion takes place at both unions, the distal one being perfect; no enterolith or distention whatever.

In the next and last specimen to which $I$ wish to call your attention, there is also the results of two experimental operations on the same subject, which was a small brown dog. An oblique enterorraphy, No. 25 was made October 31 st, 1892 , and two weeks later on November 14th, 1892, a cholecystoenterorraphy, No. 26 was made, the gall bladder being united to the duodenum. Four months later on March 13th, 1893, this animal was killed and the specimens removed as you here see them; they have been examined frequently and the unions have stood the test of thorough manipulation. This I deem a very interesting specimen and I hope you will all examine it carefully. It is probably the first time in the annals of surgery that union of the gall bladder with any part of the intestine has been made, experimentally or otherwise, wherein but two knots were applied in the suture.

In conclusion, $I$ wish to ask your consideration for the advantages which this suture offers in comparison with others:

As to the question of speed, the only operation with which this method can be compared is that of the anastomotic union by means of plates, rings or buttons; the plate method as first devised by me had six knots to tie, which was modified by Prof. Senn, he reducing the number to four; even then, in the hands of a skillful surgeon, I think this suture would compare favorably as to time.

Another point in favor of this suture, and a valuable one, is its applicability or adaptability to any part of the intestinal canal, and in whatever form you choose; you are not limited to an anastomosis, for you can make a terminal, circular or oblique 
enterorraphy with as great rapidity. In its use you are not confined to intestinal unions alone, for you can unite the gall-bladder or stomach with the intestine at any point.

You are not restricted as to the length of the incision, and this being the case the suture is very applicable in pylorectomy, whereby you can remove any length of the pyloric end of the stomach, and unite it with the duodenum by making an incision in its convex surface of the desired length; or the pyloric end of the stomach and duodenum may be cut obliquely, and union then made by this suture.

An additional great advantage which this suture has above all others, is that no foreign substance is used. While the dog will not object to bone, rawhide or brass, the human subject might, and the surgeon has always in his pocket case the material to make a union such as I have described.

And last but not least, the result obtained in these experiments forced upon me the conclusion that Czerny and Lembert's teaching that but two coats of the intestine should be included in any one suture is so far as dogs are concerned not absolutely necessary, for you will observe that I have in every case penetrated the mesenteric coat, gone into the lumen of the bowel, brought the needle out again, from the lumen of the bowel through all coats of the intestine, thereby exposing the part penetrated by the needle point to infection, by the drawing of the suture material through the bowel walls. I am satisfied that this result could not have been obtained, were it not that the point through which the thread passes is buried in the tissues and surrounded by contact of serous surfaces.

That this suture is practical, and that the results are equally as satisfactory as any union heretofore advanced, has been fully shown by the specimens submitted for your examination.

Dr. Oviatr, Wisconsin-The ideal suture is one which does away with a foreign body of any kind and we have this in Dr. Connell's stitch. Another advantage it offers is that we always have the materials at hand-catgut and a darning needle will answer the purpose. I have used Connell's stitch twenty-five times on dogs with two deaths, both of which were due to accidental causes. I think the next serious criticism to be offered the use of plates and the button is the inability to get an opening of sufficient size. I have done Connell's operation in less than ten minutes.

Dr. Herrick, Ohio-I wish to mention an interesting case of my own. A woman 48 years of age had evident symptoms of closure of the cystic duct. I performed an operation upon the gall bladder by bringing it up to the incision in the abdominal wall and holding it firmly against the abdominal walls to prevent the passage of bile into the abdominal cavity. I removed sixteen stones and washed the bladder out with warm water. I then found another accumulation in the cystic duct and removed them, closed the opening into the duct and elosed together the openings in the oladder and in the abdominal wall. I secured immediate complete union and the patient was fully recovered in two weeks.

Dr. MURPhY - I wish to thank the gentlemen for the interest they have taken in my subject. I wish to say a few words in regard to the foreign body element. The suture has been used an enormous length of time in all ways. I have not yet tried Connell's suture but am favorably impressed with it. It has been found that in the vast experience of the past the use of the suture method has resulted in a mortality of 47 per cent., while with foreign bodies the death rate is 24 per cent. I don't care what it is, the approximation that will give that kind of a result is what we want.

E. W. Axprews, Chicago-I should like to ask Dr. Connell if this lateral anastomosis is preferred by him to the end to end anastomosis.

Dr. Connell-In reply to Dr. Andrews I will say that I don't think this is the best, but it is the easiest to make. The other is nearest the ideal when the corners are satisfactorily turned in and the knot buried.

THE MURPHY BUTTON.

Dr. J. B. Murphy of Chicago, made a demonstration on a dog, of the method of making intestinal anastomosis by the use of the "button" bearing his name, after which he made a brief explanation of his method.

DR. H. O. WALKER, Michigan-I wish to speak particularly of Dr. Murphy's button, having used it three times on the human subject. The first case was a case of cholecystoenterostomy. The conditions at the time of the operation were very unfavorable, and the patient died the following morning. It required fifteen minutes to perform the operation. My next case was that of a woman 38 years old who was brought to me on the 26 th of last December. She was suffering from a fecal fistula in the right iliac region and was greatly emaciated. Her suffering was so intense that morphine had to be used liberally. Three attempts had been previously made to close the fistula. I made the incision, found the adhesions and completed the operation in seven minutes. The button was never seen again, probably on account of the negligence of the nurse, but the woman made a good recovery. The next one was a bad case of strangulated hernia which had gone unrecognized seven: days when it was brought to me, and at that time general peritonitis existed. I did the operation this time in six minutes, and in operations on the dog have done it in four minutes. One element in particular claimed for the button by Dr. Murphy should be well considered, that is the short duration of time required when it is used.

DR. RUTH, Iowa-I have for some time been considerably interested in intestinal work, and when Dr. Murphy's button first came out, I thought simply that it was another appliance to be tried, but upon second consideration of its claims. I fell in love with the thing. I performed my first experiment on the 10th of last January and have done many since then, and in no case has there ever developed a fistula, and in no case did the $d o g$ die as an immediate result. One point about the button which I wish to especially recommend, is the fact that no foreign substance is left permanently in the abdominal cavity. To the question, will the button pass out, I can say that it will. But even if we have to make a secondary laparotomy occasionally to recover a retained button, it nevertheless has sufficient recommendations.

\section{REPORT OF A CASE OF SUCCESSFUL END-} TO-END SUTURING OF INTESTINE.

Read in the Section of Surgery and Anatomy, at the Forty fourth Aunual Meeting of the American Medical Association.

$$
\begin{gathered}
\text { BY ERNEST T. TAPPEY, M.D. } \\
\text { DETROIT, MICH. }
\end{gathered}
$$

There is a decided division of opinion among surgeons on the subject of the methods employed in uniting different portions of intestine. Some are eager to try, if not to adopt, each new device in the way of plate, ring or button, as it makes its appearance; and others either cling to the older method of 